\title{
Review of: "Ti2N nitride MXene evokes the Mars-van Krevelen mechanism to achieve high selectivity for nitrogen reduction reaction"
}

Ke Chu

Potential competing interests: The author(s) declared that no potential competing interests exist.

In this manuscript, the authors reported on ambient electrochemical nitrogen electroreduction to ammonia over on two-dimensional (2D) Ti2N nitride MXene as an efficient and durable catalyst. The prepared Ti2NMXene catalysts were fully characterized and theoretically analyzed toward the NRR. Impressively, the best catalyst showed a very high NRR performance with an exceptional Faradic efficiency of 19.85\%, surpassing nearly all the reported NRR catalysts. In addition to high performance, Ti2N-MXene also exhibited a high electrochemical stability during long-term electrolysis and recycling test. The detailed Mars-van Krevelen (MvK) mechanism were also studied in detailed. Overall this manuscript is interesting, the characterizations were carefully performed and the results were clearly demonstrated. Therefore, I recommend the acceptance of this paper after the minor revisions, with below points to be addressed: $1 \square$ Introduction section: to put this work into broader contents, more recently MXene-based NRR catalysts can be cited: 10.1002/aenm.202103022, 10.1002/smll.202102363, 10.1039/d1ta03662a.

$2 \square$ NOx detection (NO2-, NO3- , NO or other NxOy) -NOx contamination in the used N2 feeding gas will cause a false positive result. The authors have to provide detailed analysis about the presence of NOx contaminants using chromatographic methods.

$3 \square$ It is better to offer the photograph of electrochemical setup for NRR test in Supporting Information.

$4 \square$ The performance comparison between current Ti2N-MXene and those state-of-the-art Ti2N-MXene catalysts for the NRR should be listed in a Table.

$5 \square$ Catalyst structure after stability test should be characterized, such as phase, morphology and chemical states. 